\title{
The Electronic Balance and Some Gravimetric Applications (The Density of Solids and Liquids, Pycnometry and Mass)
}

Randall M. Schoonover Mel-Sywe Hwang James Taylor Cheryl Smith

U.S. DEPARTMENT OF COMMERCE Technology Administration

National Institute of Standards and. Technology

Gaithersburg, MD 20899 



\section{NISTIR 5375}

\section{The Electronic Balance and Some Gravimetric Applications (The Density of Solids and Liquids, Pycnometry and Mass)}

Randall M. Schoonover Mei-Sywe Hwang James Taylor Cheryl Smith

U.S. DEPARTMENT OF COMMERCE Technology Administration

National Institute of Standards and Technology

Gaithersburg, MD 20899

February 1994

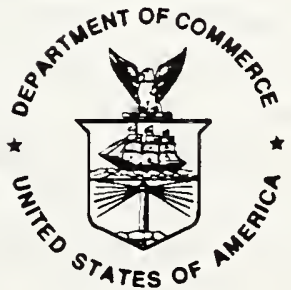

U.S. DEPARTMENT OF COMMERCE Ronald H. Brown, Secretary

TECHNOLOGY ADMINISTRATION

Mary L. Good, Under Secretary for Technology

NATIONAL INSTITUTE OF STANDARDS

AND TECHNOLOGY

Arati Prabhakar, Director 



\title{
The Electronic Balance and Some Gravimetric Applications (The density of solids and liquids, pycnometry and mass.)
}

\author{
Randall Schoonover \\ Supervisor \\ NIST \\ Gaithersburg, MD 20899 \\ Mei-Sywe Hwang \\ Statistician \\ Center for Measurement Standards \\ ITIR \\ Hsinchu, Taiwan, R.O.C.
}

\author{
James Taylor \\ Retired \\ NIST \\ Gaithersburg, MD 20899 \\ Cheryl Smith \\ Chemist \\ Aluminum Company of America \\ Alcoa Technical Center \\ Alcoa Center, PA 15069
}

\author{
KEYWORDS \\ Calibration, Hydrostatic Weighing, Pycnometry, \\ Density Measurement, Electronic Balance, Mass, Glassware
}

\begin{abstract}
In recent years the electronic force balance has been perfected to a degree that it can replace the mechanical balance in both precision and capacity. Hence, the mechanical balance is rapidly disappearing from the scene. The work reported here describes the use of the electronic balance in some high precision gravimetric applications. The balance has been examined from the user's viewpoint and its use is illustrated in measuring solid and liquid densities and mass. The density assigned to a silicon crystal is in good agreement with its accepted value to within $2.4 \mathrm{ppm}$. Likewise, the water density measurements substantiate Kell's equation for the density of water neär 23 degrees Celsius.
\end{abstract}

\section{INTRODUCTION}

Beginning in 1965 Bowman and Schoonover published a series of papers $[1,2,3,4,5]$ that described the use of the mechanical one-pan two-knife balance in the high precision determination of the density of a solid object. That work culminated in a silicon crystal density standard accurate to two parts per million (ppm). In recent years the electronic force balance [6] has been perfected to a degree that it can replace most mechanical balances in both precision and capacity. Hence the mechanical balance is rapidly disappearing from the scene. Contacts with the metrology community have convinced the authors of the need to revisit the high-precision density measurement and to extend the scope to include other applications. This work examines the electronic balance application from the user's viewpoint and discusses its use in measuring both solid and liquid density, pycnometer volume, glassware 
calibration and mass. We present measurement results of the mass and density of a $200 \mathrm{~g}$ silicon crystal and the density determination of water once distilled from a continuous flow.

\section{PRINCIPLES AND APPLICATION}

The gravimetric applications mentioned above are not disparate topics and we begin with the examination of the hydrostatic weighing principles. In particular the density determination of a solid object is discussed in simple form and later extended to a more general form. The following discussion is appropriate to all of the above applications.

\section{The Density of a Solid by Hydrostatic Weighing.}

It was shown in the 1967 work [2] that one does not need to appeal directly to a mass standard to measure the density of a solid object by hydrostatic weighing. The only requirements are knowledge of the density of air, the density of water and a linear gravimetric force scale. For completeness we present the development of the equation required for a solid object density determination when the object is suspended from a gravimetric force detector, first in air and then in water. We have chosen a simple but perfectly linear spring scale (force-balance) as our detector. To make this concept clear we begin with a special case where the temperature of the air, the water and the object are in equilibrium with each other. Furthermore, the air density and spring constant remain unchanged throughout the measurement and the detector scale reads zero when the pan is empty. Figure 1 illustrates the four detector observations required to weigh an object in air and then in water. The following two equations are expressed in terms of the observables, the known and unknown components:

$$
\begin{aligned}
& M_{x} g\left(1-\frac{\rho_{a}}{\rho_{x}}\right)=K O_{A L} \text { (air weighing) } \\
& M_{x} g\left(1-\frac{\rho_{w}}{\rho_{x}}\right)=K O_{w L} \text { (water weighing) }
\end{aligned}
$$

where $M_{x}$ is the mass of object $\mathrm{X}, \rho_{a}$ is the air density, $\rho_{w}$ is the water density, $K$ is the spring constant, $g$ is the local acceleration of gravity and $O_{\mathcal{A}}$ and $O_{w L}$ are the detector observations when loaded in air and water respectively.

Solving the above equations for the density of object $\mathrm{X}, \rho_{\mathrm{g}}$, one obtains the following equation:

$$
\rho_{x}=\frac{O_{w L} \rho_{a}-O_{A L} \rho_{w}}{O_{w L}-O_{A L}}
$$

The reader should note that the mass of $\mathrm{X}, M_{x}$, does not appear in the solution.

The caveat associated with the use of eq. (3) is the required thermal equilibrium and the constant air density during the weighing cycles. Equilibrium is nearly impossible to achieve in practice and 

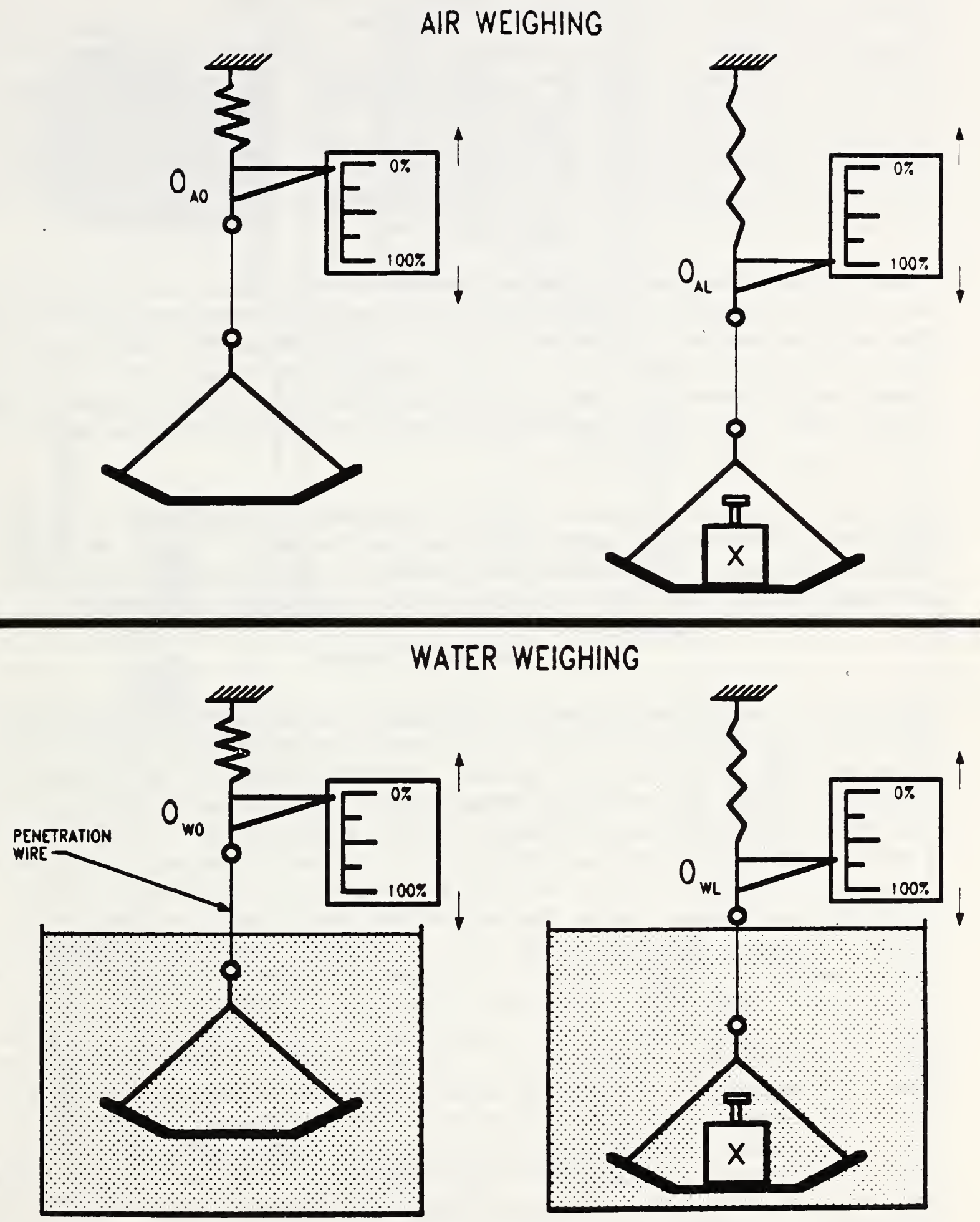

Figure 1. The spring balance is used to illustrate the principal of hydrostatic weighing. 
therefore it is desirable to have the water temperature cooler (by about $1^{\circ} \mathrm{C}$ ) than the surrounding air Otherwise water vapor is driven to the cooler balance mechanism where it condenses causing a loss of measurement precision. However, equilibrium can be closely approximated in a stable laboratory environment. Air density changes are related to temperature, barometric pressure, relative humidity and carbon dioxide content $\left(\mathrm{CO}_{2}\right)$. Normal $\mathrm{CO}_{2}$ variations and humidity excursions have a slight effect on air density and nature limits pressure variations to about $4 \%$. Climatic control systems readily maintain air temperature within $0.5^{\circ} \mathrm{C}$ or better. Therefore, air density variations are limited to about $10 \%$ and may be much less on a daily basis. Although the equilibrium constraint is violated. the resultant error may be acceptable to some users.

If one examines the investment of time and equipment required to implement the use of this method he may find it more expedient to determine density by measuring the dimensions of an ideal geometry and then weigh the specimen to determine its mass. If the dimensions are $25 \mathrm{~mm}$ or larger we should achieve an accuracy of about $0.1 \%$ with a good micrometer and a student scale. If more accuracy is desired, then the gravimetric technique described above can provide results to as good as $0.0005 \%$ but could be worse. For predictable performance at the part per million level a more general formulation will be required and is discussed later.

The preceding discussion demonstrates that the requirements for determining the density of a solid object are a force detector, a means to determine both the density of air and the density of water. thermal equilibrium of the constituents and a constant air density during the measurement. Before proceeding further a more detailed examination of these requirements is presented.

\section{The Force Detector}

Although not perfect like the spring-balance force detector described above. the modern electronic foice balance is a very good analogy. An overview of these instruments is given in [6]. A short summary of the principles of operation is given here. Detailed knowledge of the electronic circuits are unnecessary and may vary considerably between models. Figure 2 illustrates the basic principles of a force balance, and a representative mechanical structure is shown in Fig. 3. When a downward force is applied to the balance pan (loaded with an object) it is opposed by a magnetic force generated by the interaction of two magnetic fields. One field is generated by a permanent magnet and the other a controllable electromagnet. Usually, the magnetic force is applied through a multiplying lever and not by direct levitation. Sufficient magnetic force is generated to restore the mechanism (pan) to its unloaded position, or null point, relative to its structure as determined by a position sensor. Obviously, the device is electromechanical and we should expect errors of both types to arise in the use of these instruments. It is desirable in common weighing applications to tie the magnetic force to the unit of mass via calibration of the electronic circuit. The circuit is adjusted such that the algebraic sum of the gravitational and buoyant forces produces a balance indication approximately equal to the nominal value of the applied mass. It is common practice for high precision balances to be supplied with a mass standard whose density is about $8 \mathrm{~g} / \mathrm{cm}^{3}$ and with the mass adjusted close to the nominal value. This practice provides for a uniform response among balances to a given load at a given location. The usefulness of this mass is that it eliminates the need for a calibrated set of mass standards (weight set). In pursuing the application of eq. (3) it is unnecessary to quantify the mass in terms of any unit definition nor do we care about its density; it is merely a convenient method to restore the spring 


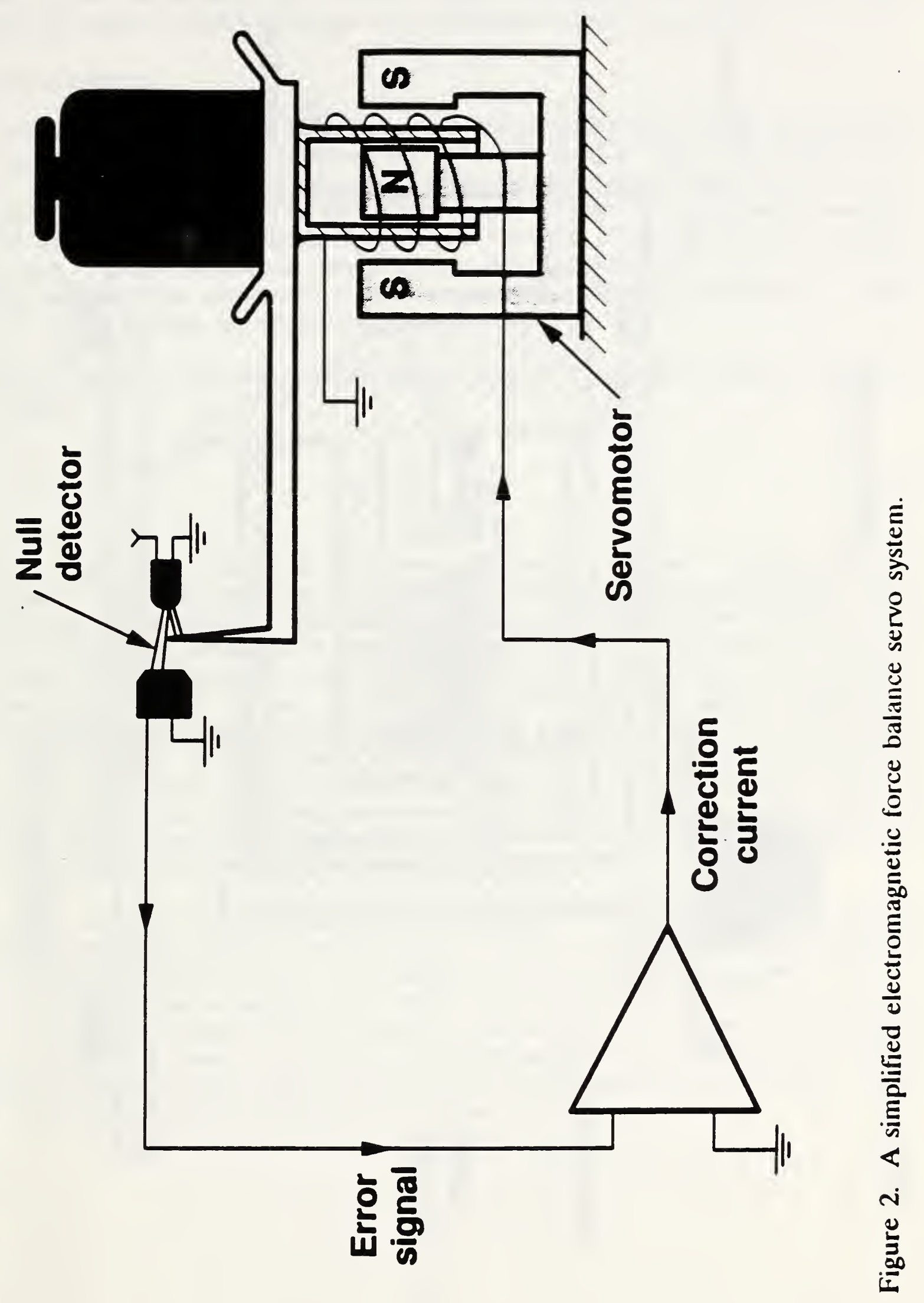




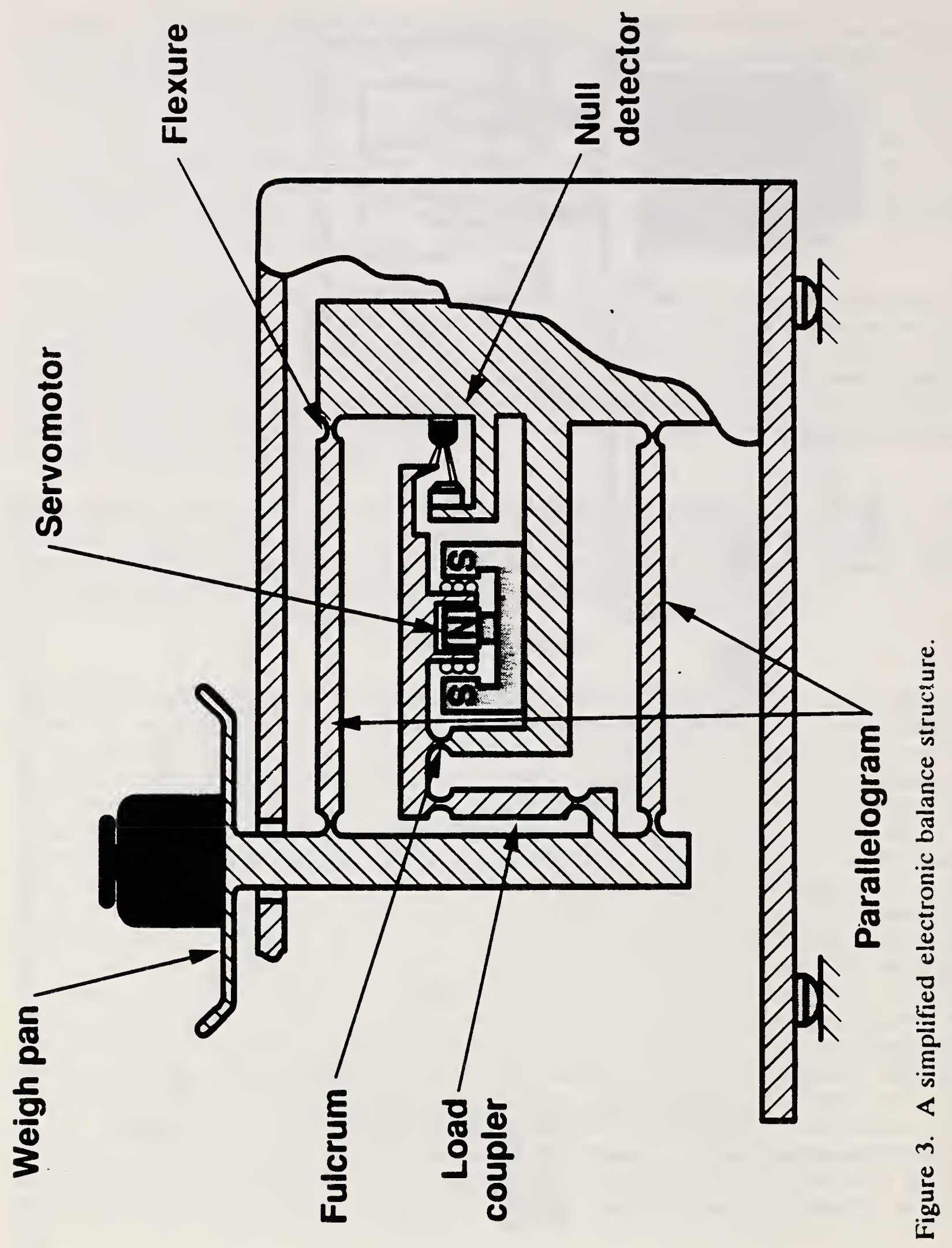


constant if shifted from the initial value. However, when the calibration weight is tied to the mass unit the electronic balance provides a convenient way to multiply and divide the mass unit.

\section{Choosing the Balance.}

A rigorous error analysis will lead to the selection of the adequate balance for the measurement at hand. An intuitive approximation is offered here and a more rigorous analysis is given later. For example, to measure the density of a $200 \mathrm{~g}$ silicon crystal two balance observations are required by the above density determination. From the air weighing equation it is obvious that the balance must have a capacity of $200 \mathrm{~g}$. The crystal density based on prior knowledge of the water density is limited to about $5 \mathrm{ppm}$. For now, assume that there is no error associated with the air density, $\rho_{a}$. The air weighing requirements are satisfied with a $200 \mathrm{~g}$ capacity balance that has a reproducability of about $5 \mathrm{ppm}$; i.e., $1 \mathrm{mg}$ or better, not a difficult requirement to meet.

Expressing the left side of the water weighing equation, equation 2, (ignoring $\mathrm{g}$ ) in terms of the crystal density yields:

$$
M_{s i}\left(1-\frac{\rho_{w}}{\rho_{s i}}\right)
$$

where $M_{s t}$ is the mass of the silicon crystal, $\rho_{s t}$ is the density of silicon, $2.3 \mathrm{~g} / \mathrm{cm}^{3}$, and $\rho_{w}$ is approximately $1 \mathrm{~g} / \mathrm{cm}^{3}$. Evaluating the above term yields a balance response of approximately $113 \mathrm{~g}$. The difference between the air and water weighings is the mass of displaced water, about $87 \mathrm{~g}$. To achieve a weighing reproducability of $5 \mathrm{ppm}$ or better on a mass of $87 \mathrm{~g}$ we need a $200 \mathrm{~g}$ capacity balance with standard deviation of $0.43 \mathrm{mg}$. Obviously, the liquid weighing imposes a more stringent requirement on the balance than does the air weighing. The requirement that both weighings be performed on the same balance leads to the selection of a $200 \mathrm{~g}$ capacity balance with a reproducabilty of $0.1 \mathrm{mg}$ for this work. If given a choice, it is preferable that the solid object just barely sink in the liquid i.e., its density is only slightly greater than the liquid density. In this example, the choice of a silicon crystal did not permit this freedom and the resulting signal-to-noise ratio, although good, has not been maximized. The balance is also exposed to the total load of the hydrostatic suspension $[2,3]$ and the submerged crystal, and the sum must not exceed $200 \mathrm{~g}$.

Furthermore, while the balance precision errors are presented here as the limiting factors in achieving our $5 \mathrm{ppm}$ measurement goal, there is another major contribution to consider. It has been assumed the balance is perfectly linear, but it is not. The manufacturer's specification for nonlinearity is $0.3 \mathrm{mg}$. For now the error is treated as random and added to $0.1 \mathrm{mg}$ reproducability for a total error of $0.4 \mathrm{mg}$. It has been implicitly indicated that the air density is without error; this is untrue but has very little effect on the choice of a balance. Although the desired measurement error $(0.43 \mathrm{mg})$ is now matched 10 a balance performance specification, this does not preclude pushing the balance to a higher level of performance, which shall be pursued later in the discussion. 


\section{Air Density}

The knowledge of the density of air and water embodies the information that ties the above density measurement to the SI units. Therefore. it is important to use the best available formulas in calculating these parameters. The air density equation for moist air used in this work is the CIPM 1981/91 recommendation [7]. This formulation ties its predecessor. CIPM-81, to the International Temperature Scale of 1990 (ITS-90) and utilizes better estimates for some of the constants and other parameters. For brevity we do not reproduce the formulation here but note that a 0.0004 mole fraction for $\mathrm{CO}_{2}$ is assumed in our laboratory.

Errors in the parameters of temperature, pressure and relative humidity do affect the uncertainty of the calculated air density. These parameters are measured with well-calibrated instruments with respective errors of $0.01{ }^{\circ} \mathrm{C}, 13$ pascals $(0.1 \mathrm{~mm} \mathrm{Hg})$, and $2 \% \mathrm{RH}$. Based on these error estimates an uncertainty of $0.0003 \mathrm{mg} / \mathrm{cm}^{3}$ has been assigned to the calculated air density values. It is this error estimate that is propagated in the analysis presented later.

\section{Water Density}

The work of Kell [8] is generally accepted as the best comprehensive treatment of water density. For the sake of brevity Kell's formula is not given here. The formula provides a value for the density of air-free water at 1 atmosphere of pressure with an estimated uncertainty of $5 \mathrm{ppm}$. The formula assumes the use of the IPTS-1968 (t68) temperature scale and temperature $t$ measured in terms of the IPTS-1990 (190) must be converted to IPTS-1968. This is readily accomplished in the range between $20{ }^{\circ} \mathrm{C}$ and $30^{\circ} \mathrm{C}$ from the following approximate relationship [9]:

$$
190-168=-0.006^{\circ} \mathrm{C}
$$

To Kell's formula is added the following terms from Bowman and Schoonover [2]:

$$
\rho_{w}=\rho_{\text {Kell }}\left[\frac{1}{1-C\left(\frac{B}{760}+\frac{I}{1033}-1\right)}\right]\left[1-\left(2.11-.053 t_{w}\right)\left(1-\frac{1}{1+D}\right)\right]\left(10^{-6}\right)
$$

Where:

$$
\begin{aligned}
& t_{w}=\text { water temperature } \\
& D=\text { days since boiling } \\
& \rho_{w}=\text { water density } \\
& C \quad=\text { compressibility }=47.7 \mathrm{ppm} / \mathrm{atm} \\
& I \quad=\text { depth of immersion, } \mathrm{cm} \\
& B \quad=\text { barometric pressure, in } \mathrm{mm} \mathrm{Hg} \\
& \rho_{\text {Rell }}=\text { Kell's water density }
\end{aligned}
$$


These terms adjust Kell's formula for the reaeration of water after boiling, dissolved gases, ar.c the sample depth (compressibility of water) respectively.

The water temperature measurements here are estimated to be uncertain by $0.003{ }^{\circ} \mathrm{C}$ with a negligible effect on the water density.

\section{A GENERAL ALGORITHM FOR HYDROSTATIC WEIGHING}

The equilibrium conditions imposed on the use of eq. (3) can be avoided with a more detailed algorithm. The chief advantage is the achievement of predictable results, i.e., an estimate of measurement uncertainty. With a little extra effort higher performance can be obtained from the balance. Both topics go hand in hand and are presented together here. However, before proceeding it is beneficial to first examine the balance usage in a simple mass measurement and how the result is affected by a nonlinear balance response.

In the above hydrostatic weighing derivation the balance response is discussed in terms of a spring constant and the product of mass and local gravity is used to adjust the constant, that is, the balance response is calibrated in terms of the gravitation force exerted on the balance. This force is expressed by the following equation:

$$
S\left(1-\frac{\rho_{a}}{\rho_{s}}\right) g=K O_{1 a}
$$

where $S$ is the mass of the calibration weight and $\rho_{s t}$ its density at temperature, $t$.

The force imposed on the balance by an object of unknown mass is as follows:

$$
M_{x}\left(1-\frac{\rho_{a}}{\rho_{x x}}\right) g=K O_{2 a}
$$

where $M_{x}$ is the mass of the unknown object and $\rho_{x x}$ is its density at temperature, t.

Therefore:

$$
S \frac{\left(1-\frac{\rho_{a}}{\rho_{s}}\right)}{L}=M_{x}\left(1-\frac{\rho_{a}}{\rho_{x}}\right)
$$

$\mathrm{L}$ is the ratio of balance observations:

$$
L=\frac{O_{1 a}-O_{\infty a}^{\prime}}{O_{2 a}-O_{\infty}}
$$


The term, $O_{o a}^{\prime}$, is the balance no-load indication during the calibration cycle and is defined to be zero and $\mathrm{O}_{\mathrm{oa}}$ is zero or near zero when the balance pan is empty during the weighing cycle. Furthermore, the balance response when the calibration weight is engaged, $O_{\perp a}-O_{o a}^{\prime}$, is redefined as $O_{c}$ for this and all remaining applicable equations. We can now express a solution for the unknown mass, $M_{x}$, in terms of the balance observations:

$$
M_{x}=\frac{S\left(1-\frac{\rho_{a}}{\rho_{s z}}\right)}{\left(\frac{O_{c}}{O_{2 a}-O_{\infty a}}\right)\left(1-\frac{\rho_{a}}{\rho_{a b}}\right)}
$$

With a knowledge of the calibration weight's mass and density at the air temperature, $t$, from a simple weighing one can calculate the mass of an unknown object. Obviously the roles of $S$ and $\mathrm{X}$ can be interchanged to perform an in siru built-in mass calibration.

In calibrating the balance, the manufacturer forces the no-load indication to be zero and when the calibration mass is engaged, forces it to indicate its nominal value. The ideal balance response is, of course, a straight line connecting these points and for some balances to extrapolate beyond these bounds. Usually, balances do not respond in the ideal manner and therefore any observation not at these points may require correction for nonlinearity. In the following discussion it is assumed that the correction for nonlinearity has been applied to the balance observations for the unknown object during both the air and water weighings. The balance linearity topic will be revisited later in the discussion.

\section{The General Hydrostatic Weighing Equations}

It has been assumed that the air densities during the air and water weighings are different and it follows that the various temperatures, barometric pressure, and relative humidity involved may also be different. Temperature variations assure that there will be two slightly different densities for the object density during the measurement sequence. Fortunately, this condition can be accounted for but it will require additional information. This does not imply that there can be a lack of thermal equilibrium between the constituents during each of the weighings but rather different temperatures may be encountered during the air and water weighing. However, as noted earlier it is desirable to have the water temperature slightly cooler than the surrounding air during the water weighing cycle. Special precautions [2] must be taken to protect the measurement from the undesirable effects of this boundary condition.

The above simple weighing equations are now replaced with ones that permit variations in the ambient conditions that surround the objects during the weighings. Furthermore, the dependency of the balance calibration with respect to air density is now taken into account. In addition, it is desirable to express the object density at a reference temperature that may be different from that of the measurement. The expanded weighing equations are: 


$$
\begin{aligned}
& S \frac{\left(\frac{1-\rho_{a a}}{\rho_{s} Z_{1}}\right)}{\left(\frac{O_{c}}{O_{2} a-O_{o b}}\right)} g=M_{x}\left(\frac{1-\rho_{a a}}{\rho_{x t n}}\right) X_{g} \quad \text { air weighing } \\
& S \frac{\left(\frac{1-\rho_{a w}}{\rho_{s} Z_{2}}\right)}{\left(\frac{O_{c}}{O_{2} w-O_{o w}}\right)} g=M_{x}\left(\frac{1-\rho_{w}}{\rho_{x t n}}\right) Y g \quad \text { water weighing }
\end{aligned}
$$

Solving the above equations for the unknown density, $\rho_{x m}$ we have:

$$
\rho_{\text {zan }}=\left[\frac{\left(1-\frac{\rho_{a s}}{\rho_{s} Z_{1}}\right)}{\left(\frac{O_{c}}{O_{2 a}-O_{\infty}}\right)} \rho_{w} Y-\frac{\left(1-\frac{\rho_{a w}}{\rho_{s} Z_{2}}\right)}{\left(\frac{O_{c}}{O_{2 w}-O_{o w}}\right)} \rho_{c o} X\right] /\left[\frac{\left(1-\frac{\rho_{a s}}{\rho_{s} Z_{1}}\right)}{\left(\frac{O_{c}}{O_{2 a}-O_{\infty}}\right)}-\frac{\left(1-\frac{\rho_{\alpha w}}{\rho_{s} Z_{2}}\right)}{\left(\frac{O_{c}}{O_{2 w}-O_{o w}}\right)}\right]
$$

where:

$$
\begin{aligned}
& \rho_{\text {m n }} \quad=\text { Density of solid under test at } 20^{\circ} \mathrm{C} \\
& S \quad=\text { Mass of calibration weight and } \rho \text {, its density at } 20^{\circ} \mathrm{C} \\
& \text { in }=20^{\circ} \mathrm{C}
\end{aligned}
$$

The reader will note that the density of the calibration weight now appears in the solution for $\rho x i n$ whereas its mass does not.

\section{Air Weighing}

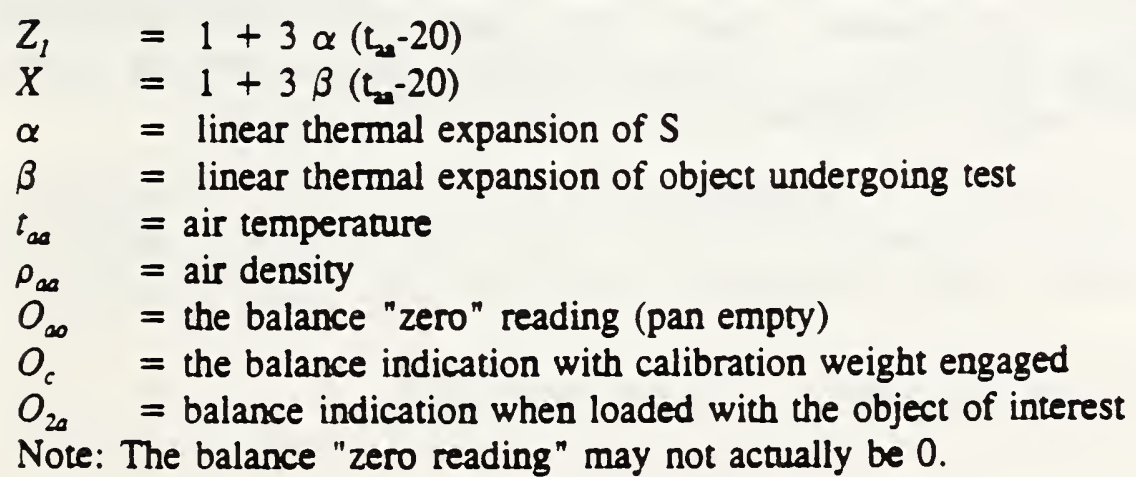




\section{Water Weighing}

$$
\begin{array}{ll}
Z_{2} & =1+3 \alpha\left(t_{A w}-20\right) \\
Y & \left.=1+3{ }_{3}-20\right) \\
t_{w} & =\text { water temperature } \\
\rho_{w} & =\text { water density } \\
t_{a w} & =\text { air temperature } \\
\rho_{a w} & =\text { air density }
\end{array}
$$

Note: $O_{2 w}$ is comparable to $O_{2 a}$ above

The differences in elevation between the calibration weight, the air weighing pan and the lower immersed pan require corrections for the gradient in the earths gravitational field $[2,10]$. This correction is approximately 200 micrograms per meter per kilogram. In the case of the balance used here the calibration weight and the air weighing pan are at the same elevation and the (lower) water weighing observations are adjusted to that elevation.

\section{Linearity Test and Correction}

The terms above, $O_{2 a}$ and $O_{2 w}$, are assumed to have been corrected for any nonlinear balance response. We obtained the corrections by observing the balance response at $25 \%, 75 \%$ and $100 \%$ of capacity. It was fortuitous that most of the loads in this work occurred at or near these points or the points of calibration. The balance is calibrated with a $100 \mathrm{~g}$ weight and its capacity is $200 \mathrm{~g}$; therefore, an additional $100 \mathrm{~g}$ and $50 \mathrm{~g}$ weight is required for the test. As noted earlier the balance calibration forces a pan-empty indication of 0.0000 and the $100 \mathrm{~g}$ indication to be 100.0000 ; these points are given and not observed. However, it is prudent to immediately check the calibration by reweighing the calibration weight or its replica. This provides assurance that an error-free calibration occurred. Repea! the procedure as necessary as some balances require several cycles to obtain a stable calibration.

The balance is used to measure the difference between the calibration weight and its replica until the difference, in terms of the calibrated balance indication, is known with insignificant error. The sum of these two weights is used to test the balance at $100 \%$ capacity and any observable difference between the ideal and observed indication is a linearity error. That is, the observed response plus the correction equals the ideal response. In a similar manner the difference between the $100 \mathrm{~g}$ calibration weight and the sum of two $50 \mathrm{~g}$ weights can be obtained. These weights should have the same nominal density as that of the calibration weight $\left(8.0 \mathrm{~g} / \mathrm{cm}^{3}\right)$. A sum and difference weighing between the calibration weight, $\mathrm{S}$, and the two $50 \mathrm{~g}$ weights, designated $\mathrm{D}$ and $\mathrm{E}$, results in two equations:

$$
\begin{gathered}
S-(D+E)=O_{1} \\
D-E=O_{2}
\end{gathered}
$$

Where $O_{1}$ and $O_{2}$ are the balance observations. The solution for $D$ is

$$
\mathrm{D}=\left(\mathrm{S}+\mathrm{O}_{2}-\mathrm{O}_{1}\right) / 2
$$


$\mathrm{D}$ is in terms of balance units as defined by the calibration weight $\mathrm{S}$. It is unnecessary to solve for $\mathrm{E}$ as it is of no further use here. In this application the balance is used to detect small differences between nearly identical loads and the effect of any balance nonlinearity is trivial. Obviously we can measure the above differences a number of times and minimize the the balance imprecision or use a more precise balance. Similarly we could perform the linearity test multiple times to decrease the correction uncertainty. We discuss this aspect of the measurement in the analysis section of the paper. The linearity measurement sequence expressed as a percentage of capacity is:

\section{$\begin{array}{lllllllllllllllll}0 & 25 \% & 50 \% & 75 \% & 100 \% & 75 \% & 50 \% & 25 \% & 0\end{array}$}

The corresponding observations are:

$$
\begin{array}{llllllllll}
\mathrm{O}_{1} & \mathrm{O}_{2} & \mathrm{O}_{3} & \mathrm{O}_{4} & \mathrm{O}_{5} & \mathrm{O}_{6} & \mathrm{O}_{7} & \mathrm{O}_{3} & \mathrm{O}_{9}
\end{array}
$$

This sequence minimizes the effects of drift and hysteresis, if any, and can be abbreviated to include only the required range or just one point. In the manner described above additional weights could be used to obtain the $12.5 \%$ point and so on. The response of our balance is depicted graphically by Fig. 4. We note the 0 and $50 \%$ points are forced to be on the ideal response line and that the others may not be. The "zero" observations are only necessary to insure that the balance is in the zero caprure range of the mechanism and preferably the pan is nearly empty. If not, the user may inadvertently shift the origin of the curve between measurements and not be able to characterize the response on a long term basis. For this reason it is wise to disconnect the immersed weighing pan before performing the balance calibration during the water weighing cycle. Thereafter the balance is not rezeroed. Additionally, the balance should always be leveled before use.

The linearity correction is derived relative to the calibration weight $S$, here the $50 \%$ point. We obtain the correction at the $75 \%$ point by subtracting the average of observations $\mathrm{O}_{3}$ and $\mathrm{O}_{7}$ from the average of $\mathrm{O}_{4}$ and $\mathrm{O}_{6}$. This difference plus the linearity correction, $\mathrm{Lc}$ is equated to the value obtained for weight $\mathrm{D}$ above :

$$
\mathrm{D}=\left(\left(\mathrm{O}_{4}+\mathrm{O}_{6}\right) / 2\right)-\left(\left(\mathrm{O}_{3}-\mathrm{O}_{7}\right) / 2\right)+\mathrm{LC}
$$

Similar expressions can be written for the other points and the corresponding linearity correction can be obtained for each one.

\section{LIQUID DENSITY BY HYDROSTATIC WEIGHING}

An examination of eq. (13) reveals the possibility of determining the water density, or the density of other liquids, with prior knowledge of the solid object density. Relabeling the terms $\rho_{w}$ as $\rho_{L}$ and $\rho_{x}$ as $\rho_{s t}$ and rearranging terms, the density of an unknown liquid by hydrostatic weighing is: 


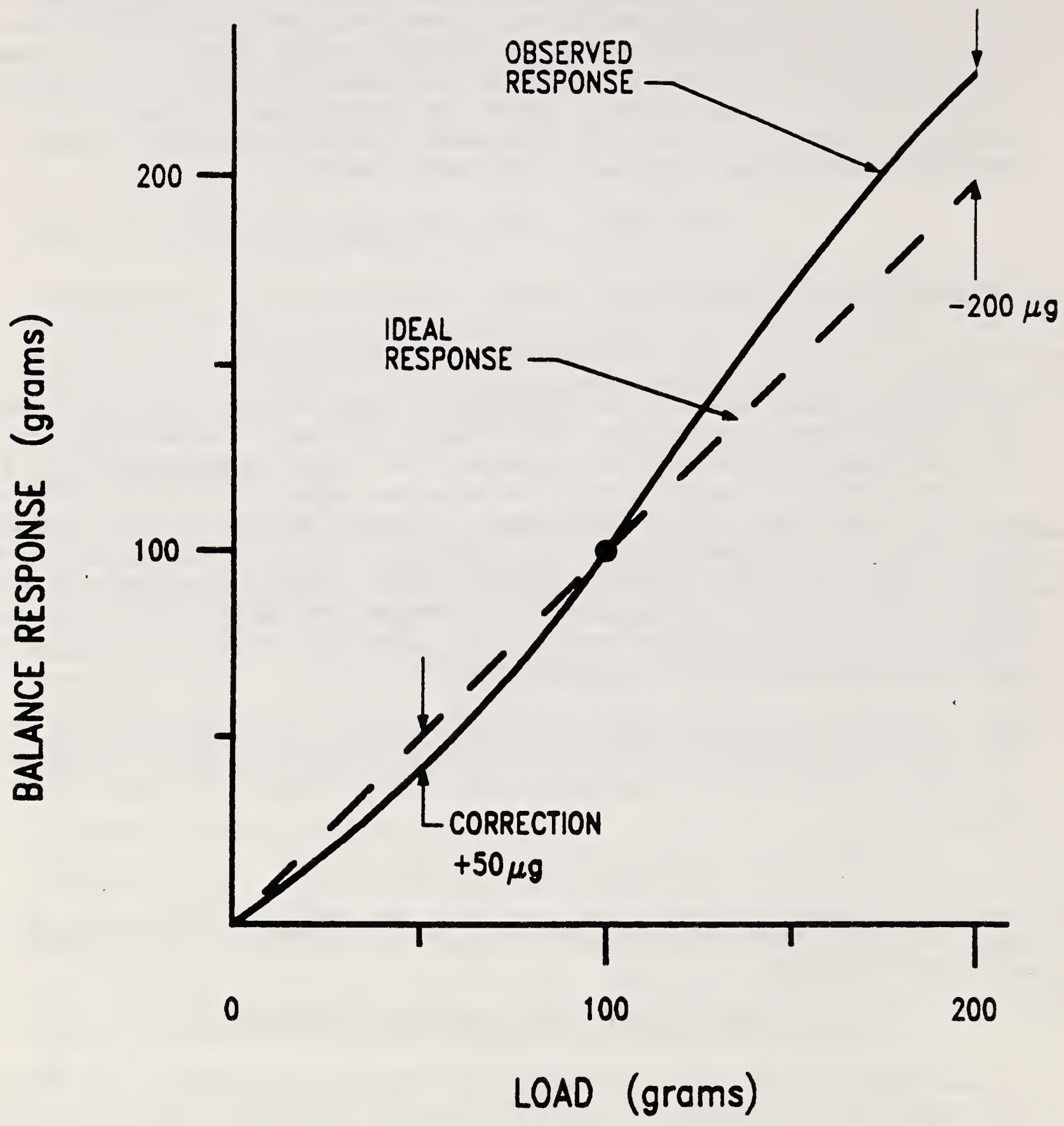

Figure 4. The balance response illustrated here is typical of the model used for this work. 


$$
\rho_{L B t}=\left[\rho_{S i t n}\left(\frac{\left(1-\frac{\rho_{a a}}{\rho_{s} Z_{1}}\right)}{\left(\frac{O_{c}}{O_{2 a}-O_{o a}}\right)}-\frac{\left(1-\frac{\rho_{a w}}{\rho_{s} Z_{2}}\right)}{\left(\frac{O_{c}}{O_{2 w}-O_{o w}}\right)}\right)+\rho_{a a} X \frac{\left(1-\frac{\rho_{a L}}{\rho_{s} Z_{2}}\right)}{\left(\frac{O_{c}}{O_{2 w}-O_{o w}}\right)}\right] / Y \frac{\left(1 \frac{\rho_{a a}}{\rho_{s} Z_{1}}\right)}{\left(\frac{O_{c}}{O_{2 a}-O_{o a}}\right)}
$$

where:

$\rho_{L B t}=$ Liquid density under test at the bath temperature, $\mathrm{Bt}$.

$\rho_{s t}=$ Solid object density standard normalized at $25^{\circ} \mathrm{C}$.

in $=25^{\circ} \mathrm{C}$

\section{PYCNOMETER CALIBRATION}

The pycnometer is a vessel that can be used to determine the density of liquids and solids, see figure 5. The discussion is here confined to the liquid density application. In practice the user must know the pycnometer capacity. This is readily accomplished by weighing the pycnometer empty and then filled with pure water at a known temperature. From the two weighings and with knowledge of water density, the pycnometer capacity can be calculated. However, examination of the details of a pycnometer calibration reveals a minor difficulty. That is, the pycnometer must be soaked in a temperature-controlled bath to measure the temperature of the contained water. It is assumed that with sufficient thermal soaking all constituents obtain the bath temperature. However, when the pycnometer is removed from the bath for weighing, the exterior must be dried before storing in the balance over night to achieve thermal equilibrium [11]. At the time of weighing the total displacement volume, pycnometer and contents, may have changed from the initial condition of the water bath, i.e., a cnaree in temperature. The capacity of interest is at the temperature of the water bath while the buoyant force at the time of weighing depends on the balance temperature.

The pycnometer capacity, C, at the bath temperature is given by the solution of the empty and full weighings (not shown) with correction for the change in displacement volume above:

$$
\begin{gathered}
\left.C_{B E}=\left[S\left(\frac{\left(1-\frac{\rho_{a F}}{\rho_{s} Z 2}\right)}{\left(\frac{O_{c}}{O_{2 F}-O_{o F}}\right)}-Q \frac{\left(1-\frac{\rho_{a E}}{\rho_{S} Z 1}\right)}{\left(\frac{O_{c}}{O_{2 E}-O_{o E}}\right)}\right)\right] /\left[1-\frac{\rho_{o F}}{\rho_{W F}}\right) \rho_{W B t}\right] \\
Q=\frac{\left(1-\left(\frac{\rho_{a F}}{\rho_{P}}\right) Y\right.}{\left(1-\left(\frac{\rho_{a E}}{\rho_{P}}\right) X\right)}
\end{gathered}
$$




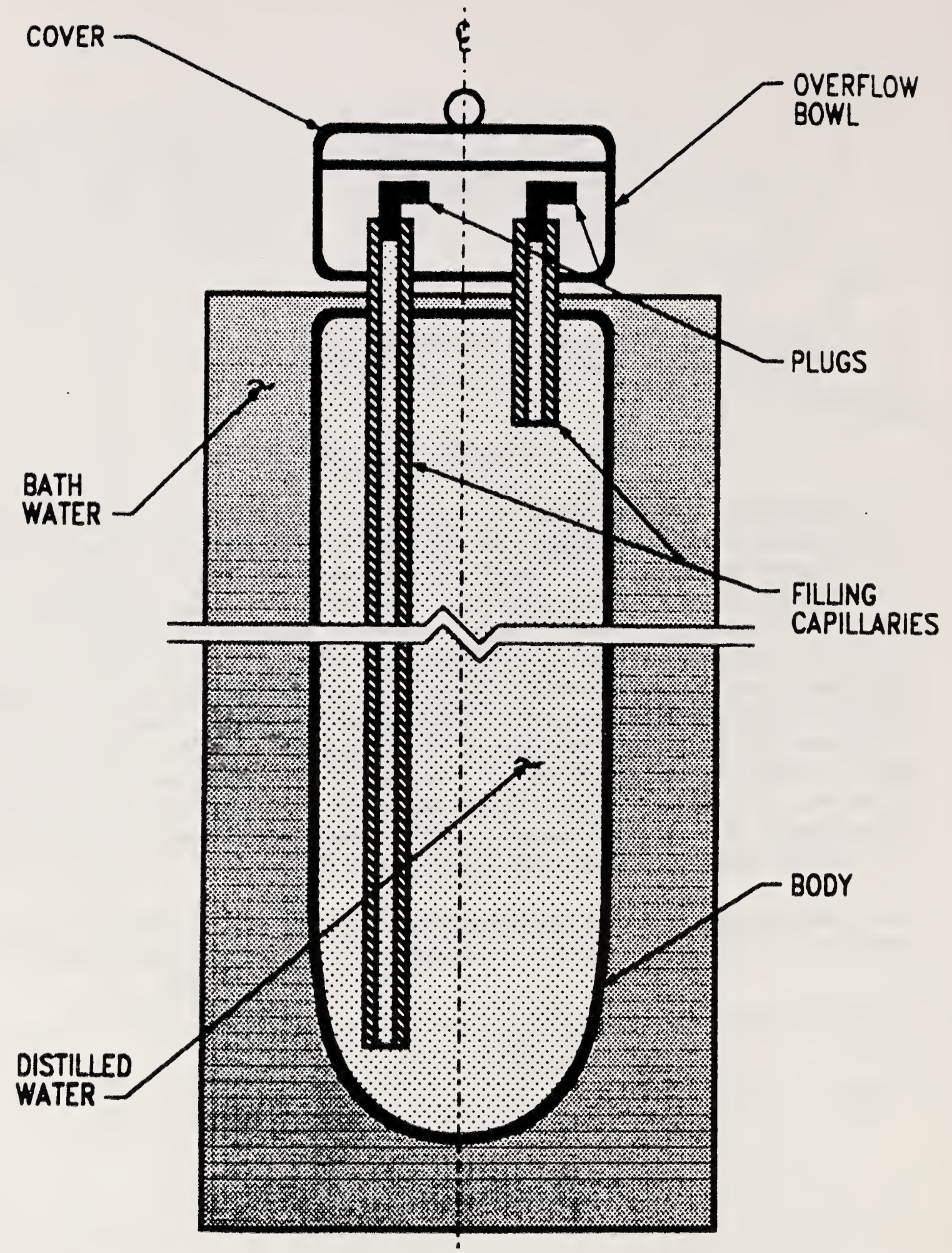

Figure 5. A schematic illustration of the pycnometer immersed in the constant temperature bath. For clarity the plugs are shown partially inserted. 
where:

$\rho_{n F}=$ Water density during the air weighing of the full pycnometer

$\rho_{a F}=$ air density during full weighing

$\rho_{a E}=$ air density during empty weighing

$\rho_{p}=$ the approximate density of the pycnometer body at $20^{\circ} \mathrm{C}$

The mass of the calibration weight, $S$, now appears in a measurement solution for the first time other than the simple mass measurement of eq. (10).

The pycnometer design accommodates any water volume expansion with its covered overflow bowl, see Fig. 5. It was designed at NIST years ago, with the wire plugs shown in Fig. 5. added for this work. These stoppers make it unnecessary to set the water meniscus at the ends of the filling capillaries.

It is noteworthy that weighing glass pycnometers in low humidity environments can be plagued with static charge. Static charge can be detected with an inexpensive capacitance based electrometer. As a precaution the exterior surface was coated with a slightly visible amount of tin oxide to prevent charge and none was detected during its use. At this time the pycnometer measurement data has not been analyzed and will be presented in a later report.

\section{PYCNOMETER APPLICATION}

With the pycnometer volume (capacity) assigned by the above method it can be used in a similar fashion to determine the density of other liquids. The error associated with the water calibration will be carried forward in its use to determine the density of other liquids. It follows from the solution of the empty and full pycnometer weighings that the unknown liquid density, $\rho_{L B}$, at the bath temperature, $\mathrm{Bt}$ is:

$$
\begin{aligned}
\rho_{L B E} & =\left[S\left(\frac{\left(1-\frac{\rho_{a}}{\rho_{s} Z_{2}}\right)}{\left(\frac{O_{c}}{O_{2 F}-O_{o F}}\right)}-R \frac{\left(1-\frac{\rho_{a E}}{\rho_{s} Z_{1}}\right)}{\left(\frac{O_{c}}{O_{2 E}-O_{o E}}\right)}\right)\right] /\left[\left(C_{2 s}\left(1+k\left(B_{t}-25\right)\right)+\rho_{a F}\left(\frac{\rho_{L B t}}{\rho_{L F t}}\right)\right]\right. \\
R & \left.=\left[\left(1-\frac{\rho_{a F}}{\rho_{P}}\right) X\right] / /\left(1-\frac{\rho_{a E}}{\rho_{P}}\right) Y\right]
\end{aligned}
$$

where:

$C_{25} \quad=$ pycnometer volume at $25^{\circ} \mathrm{C}$

$\rho_{L f}=$ the liquid density during the weighing.

$k \quad=$ cubic thermal coefficient of pycnometer body 
The problem of filling the pycnometer at the bath temperature and weighing it at another requires pricr knowledge of the density ratio, $\rho_{L B r} / \rho_{L f f}$. In practice one can adjust these temperatures close to each other and eliminate the teim. If not, handbook information may be available or the liquid dilation may be determined with additional pycnometer measurements and appropriate iteration.

\section{GLASSWARE CALIBRATION}

Flasks, burettes and pipettes are usually calibrated by gravimetric means and it is a simple matter to apply the pycnometer calibration equation above to this application. However, in general glassware does not warrant such attention to detail. A simplified equation is offered here for the calibration of general use glassware. The capacity, $C_{t}$, is:

$$
C_{t}=s \frac{\left(1-\frac{\rho_{a}}{\rho_{s}}\right)\left(\frac{0_{F}-O_{E}}{O_{c}}\right)}{\rho_{w}-\rho_{a}}
$$

Where:

$t \quad=$ water temperature in degrees Celsius

$\rho_{a} \quad=$ average air density of the weighing cycles

$\rho_{w} \quad=$ water density

$\rho_{s} \quad=$ calibration weight density at room temperature

$O_{E} \quad=$ empty flask balance indication

$O_{F} \quad=$ flask plus water balance indication

In the above equation it is assumed that the balance has been zeroed before the empty and full weig! ?. ?gs and the flask capacity is at the temperature of the water. The nominal value of the built-in calibrarion mass, $S$, is usually adjusted to be accurate within the least significant digit displayed by the balance and may not need additional calibration for this application. It has been assumed the weight is made from a material with a density near $8.0 \mathrm{~g} / \mathrm{cm}^{3}$ and has been adjusted to the "8.0 apparent mass scale".

\section{ANALYSIS}

The method of $\mathrm{Ku}[11]$ has been used to propagate errors in the functional relationship, $f\left(X_{1}, X_{2}, \ldots, X_{1}\right)$, of the uncorrelated variables $X_{1}, X_{2}, \ldots, X_{n}$. Tables $1-5$ present for each variable its value, the estimated standard deviation and an evaluation of the partial derivatives. At the bottom of each table is the estimated combined standard deviation for the function as given by the following relationship:

$$
(S D)^{2}=\sum_{i=1}^{n}\left(\frac{\partial f}{\partial X_{i}}\right)^{2}\left(S D_{i}\right)^{2}
$$



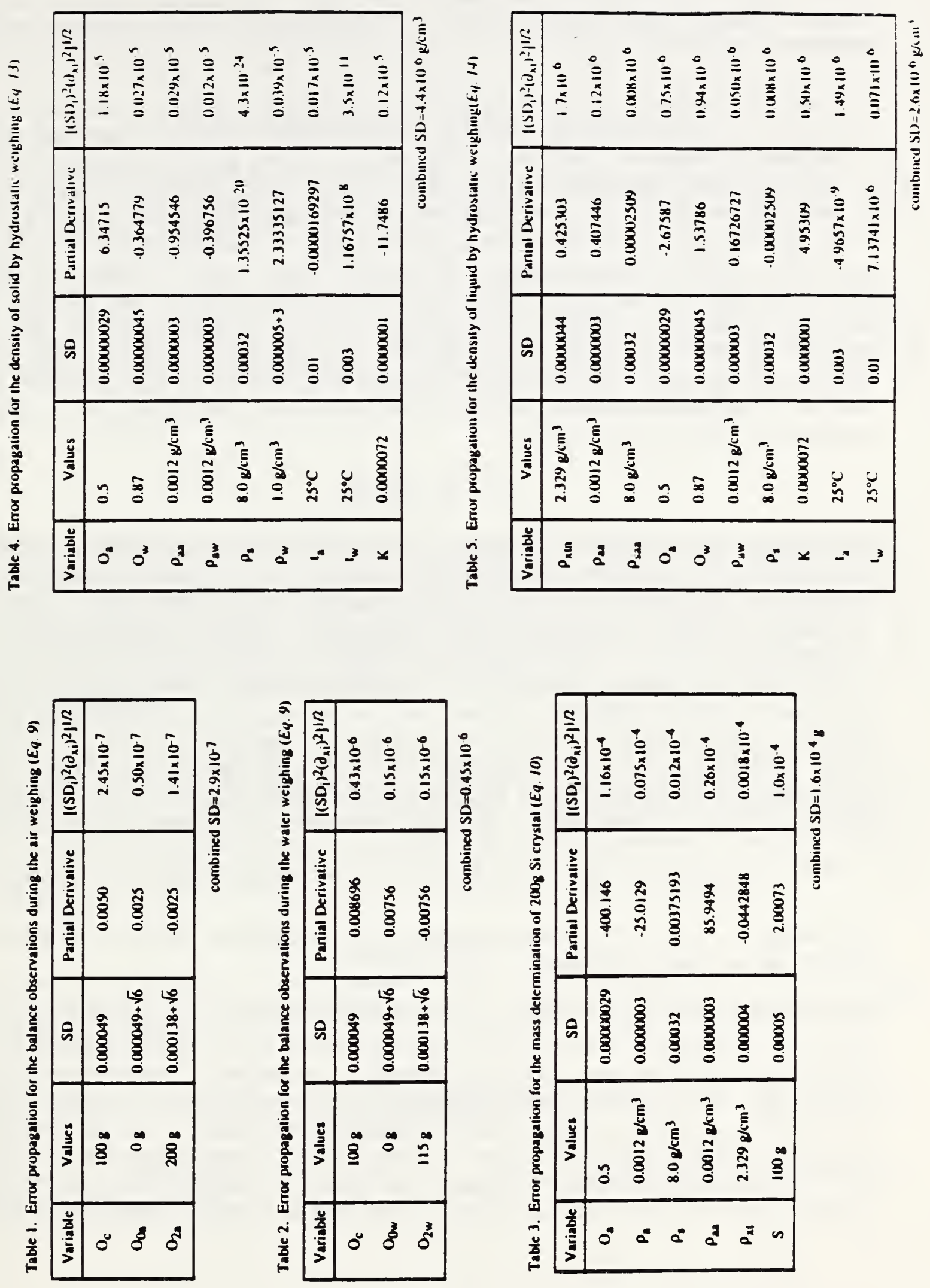
In this work, the measurement uncertainty for the mass of the silicon crystal, its density, and the measured density of water, is defined to be 3 times the combined estimated standard deviation from tic appropriate table.

An important parameter in the error analysis is the balance reproducabilty as measured by the standard deviation. The balance used here, like many electronic balances, performs better when lightly loaded. Its standard deviation was found to be $49 \mathrm{ug}$ from 0 to $100 \mathrm{~g}$ and $118 \mu \mathrm{g}$ upward to $200 \mathrm{~g}$. These standard deviations are combined in quadrature with the standard deviation of the linearity correction. At the $200 \mathrm{~g}$ level of the air weighings the combined standard deviation is $138 \mu \mathrm{g}$, while water weighings near $100 \mathrm{~g}$ are nearly free of nonlinearity and the standard deviation remains at $49 \mu \mathrm{g}$. As previously noted the balance weighings were repeated 6 times, which is reflected in Tables 1 and 2 . It is noteworthy that the balance calibration reproducabilty $(49 \mu \mathrm{g})$ is not improved by repeated calibration cycles and therefore is only performed once. This standard deviation cannot be obtained explicitly but from the nature of digital circuits it is known to be less than $1 / 2$ a count i.e. $50 \mu g$ and the value $(49 \mu \mathrm{g})$ determined by repeated weighings at the $100 \mathrm{~g}$ level was used here. Furthermore. the ratio of the balance indications is dimensionless and it is the standard deviation of the ratios that are propagated in the remaining tables.

\section{DATA}

As described earlier the balance weighings were repeated six times and defined as one measurement. In addition five independent determinations of each property, i.e, mass, crystal density, and water density were performed. For example, each time the silicon crystal density was measured by hydrostatic weighing the distilled water was replaced and the immersed constituents degassed by boiling the apparatus in situ [2]. The estimates of the balance standard deviation were obtained from the within-group of six repeated measurements. The weighing sequence used in the collection of these data began with a balance calibration followed with six pan-empty and pan-loaded weighings using. symmetrical format. This sequence results in 13 balance observations: A1 B1 A2 B2 A3 B3 A4 [uب A5 B5 A6 B6 A7 where the A's correspond to the empty pan observations and the B's to the loaded pan observations. This measurement sequence was immediately followed with the balance linearity test described earlier. The preceding six weighings are reduced to an average value as follows:

$$
[(\mathrm{B} 1+\mathrm{B} 2+\mathrm{B} 3+\mathrm{B} 4+\mathrm{B} 5+\mathrm{B} 6) / 6]-[(\mathrm{A} 1-\mathrm{A} 2-\mathrm{A} 3-\mathrm{A} 4-\mathrm{A} 5-\mathrm{A} 6-\mathrm{A} 7) / 7]=\text { Average }
$$

The linearity correction for a balance indication of B is then applied. The "A" values are always near zero and do not require correction.

The methods reported here are demonstrated with measurements of the mass, and density of a $200 \mathrm{~g}$ silicon crystal and the density of water. The silicon crystal mass was determined by an independent comparison to standards on a more precise balance by the method of combinational weighing. The average mass value assigned by intercomparision to mass standards is $199.42655 \mathrm{~g}$. The difference between this mass and that obtained by direct weighing on the balance is $0.07 \mathrm{ppm}$ and their respective uncertainties are $0.25 \mathrm{ppm}$ and $2.25 \mathrm{ppm}$. The silicon crystal density is known to be $2.3291226 \mathrm{~g} / \mathrm{cm}^{3}$ at $20{ }^{\circ} \mathrm{C}$ [5] with an uncertainty of $2 \mathrm{ppm}$. The mean value assigned from these measurements is $2.329117 \mathrm{~g} / \mathrm{cm}^{3}$ at $20^{\circ} \mathrm{C}$., a difference of $2.4 \mathrm{ppm}$ with an estimated uncertainty of $5.7 \mathrm{ppm}$. The 
measured water density values differ from those calculated by the Kell equation with corrections for dissolved gasses and depth of immersion as follows:

$$
\text { Kell - measured values }=6,0,-1,0,7 \mathrm{ppm}
$$

The water temperature ranged from $23.115^{\circ} \mathrm{C}$ to $23.516^{\circ} \mathrm{C}$. The estimated uncertainty of the values assigned here is $3.4 \mathrm{ppm}$. Given the $5 \mathrm{ppm}$ uncertainty assigned to Kell's values, differences as large as $8.4 \mathrm{ppm}$ could be expected.

With the advantage of hindsight it is clear some improvement to the measurements could be obtained by not using the balance microprocessor built-in calibration function. Performing the calibration manually would permit the advantage of multiple calibrations and may result in a smaller estimated standard deviation. However, this technique would cause the inconvenience of a longer measurement sequence.

\section{CONCLUSIONS}

We believe our investigations of the testing and use of the electronic balance support the contention that very respectable measurements at the parts per million level can be achieved in the measurement areas demonstrated. It is noteworthy that when the balance is used properly the need for well-calibrated laboratory weight sets is eliminated. Most users would be well served by obtaining a mass value for the balance calibration weight from an appropriate source along with a density determination. The needs of many users would certainly be satisfied by accepting the value and tolerance assigned by a reputable balance maker.

We did not cover all of the common uses of balances. There are simple applications such as the tolerance testing of weights for regulatory use, i.e., weights and measures, where these techniques can be adopted. It appears to us that many modern electronic balances perform so well that it might be beyond the ability of many laboratories to adequately test and calibrate them. This could well be a problem in the future where some applications, requiring certification, are limited by the ability of the certifying agency.

\section{REFERENCES:}

[1] Bowman, H.A.; Gallagher, W.; Schoonover, R.M., "The Development of a Working Density Standard, "Instrument Society of America, 20th Annual ISA Conference and Exhibit, Preprint No. 14.84.65, Los Angeles, CA (1965).

[2] Bowman, H.A.; Schoonover, R.M., "Procedure for High Precision Density Determinations by Hydrostatic Weighing," J. Res. NBS 71C: 179-198 (1967).

[3] Bowman, H.A.; Schoonover, R.M.; Carroll, C.L., "A Density Scale Based on Solid Objects,: J. Res. NBS 78A: 13-40 (1974). 
[4] Bowman, H.A.; Schoonover, R.M.; Carroll, C.L., "The Utilization of Solid Objects as Refereice Standards in Density Measurements," Metrologia 10, 117-121 (1974).

[5] Bowman, H.A.; Schnonover, R.M.; Carroll, C.L., "Reevaluation of the Densities of the Four NBS Silicon Crystal Standards," NBSIR 75-768. Final report prepared for Mechanics Division, Institute for Basic Standards (1975).

[6] Schoonover, R.M., "A Look at the Electronic Analytical Balance," Anal. Chem. 973A-980A (1982).

[7] Davis, R.S.; "Equation for the Determination of the Density of Moist Air (1981/91)" Metrologia (1992), 29.

[8] Kell, G.S.; "Density, Thermal Expansivity, and Compressibilty of Liquid Water from 0 to $150^{\circ} \mathrm{C}$ : Correlations and Tables for Atmospheric Pressure and Saturation Reviewed and Expresssed on 1968 Temperature Scale" Journal of Chemical Engineering Data, Vol 20, No. 1, (1975).

[9] Magnum, B.W.; Furakawa, G.T., "Guidlines for realizing the International Temperature Scale of 1990 (ITS-90), " NIST Technical Note 1265.

[10] Almer, H.E; Swift, H.F.; "Gravitational Configuration Effect Upon Precision Mass Measurements," Rev. Sci Instrum., Vol. 46, No. 9, September (1975).

[11] Schoonover, R.M.; Taylor, J.E., "Some Recent Developememnts at NBS in Mass Measurements," IEEE Instrumentation and measurement Conference, Boulder, CO (1986)

[12] Ku, H.S.; "Statistica! Concepts in Metrology" Handbook of Industrial Metrology, Chapter 3 , American Society of Tool and Manufacturing Engineers, Prentice-Hall, NY, (1967). 
,

. 
